Bull. Austral. Math. Soc.

$46 \mathrm{~B} 22$

VOL. 66 (2002) [313-316]

\title{
A SUBDIFFERENTIAL CHARACTERISATION OF BANACH SPACES WITH THE RADON-NIKODYM PROPERTY
}

\author{
J.R. GILES
}

\begin{abstract}
A Banach space has the Radon-Nikodym Property if and only if every continuous weak ${ }^{\star}$ lower semi-continuous gauge on the dual space has a point of its domain where its subdifferential is contained in the natural embedding.
\end{abstract}

Collier [3, Theorem 1, p. 103] proved that a Banach space $X$ has the RadonNikodym Property if and only if every continuous weak* lower semi-continuous convex function on an open convex subset $A$ of the dual $X^{\star}$ is Fréchet differentiable at the points of a dense $G_{\delta}$ subset of $A$. Recently, Bachir and Daniilidis [1, Theorem 1, p. 379] extended this result to prove that $X$ has the Radon-Nikodym Property if and only if every continuous weak ${ }^{\star}$ lower semi-continuous convex function defined on $X^{\star}$ is Gâteaux differentiable at some point of its domain with derivative in the natural embedding $\widehat{X}$. This result of Bachir and Daniilidis is related to a Gâteaux differentiability characterisation of Asplund spaces, [6, Theorem II.2, p. 9], [4, Theorem 2, p. 268]. However, Asplund spaces can also be characterised by a property of the subdifferential mapping of its continuous gauges, [5, Theorem 2, p. 155]. It is possible, following this approach, to provide a characterisation of Banach spaces with the Radon-Nikodym Property by subdifferentials of continuous weak ${ }^{\star}$ lower semicontinuous gauges, a simpler proof of the result of Bachir and Daniilidis and an extension of our characterisation of Asplund spaces.

For our proof we use the following characterisation, $[2$, Corollary $3.7 .6,(1) \Longleftrightarrow$ (3), p. 67].

Proposition. A Banach space $X$ has the Radon-Nikodym Property if and only if every closed bounded convex subset of $K$ of $X$ contains an extreme point of $\widehat{\widehat{K}}^{w^{\star}}$.

For a continuous gauge $p$ on a Banach space $X$, the subdifferential of $p$ at $x_{0} \in X$ is a nonempty weak ${ }^{\star}$ compact convex subset of $X^{\star}$,

$$
\partial p\left(x_{0}\right) \equiv\left\{f \in X^{\star}: f\left(x_{0}\right)=p\left(x_{0}\right) \text { and } f(x) \leq p(x) \text { for all } x \in X\right\}
$$

and $p$ is Gâteaux differentiable at $x_{0}$ if and only if $\partial p\left(x_{0}\right)$ is singleton, $([7$, p. 5]).

Received 2nd April, 2002

Copyright Clearance Centre, Inc. Serial-fee code: 0004-9727/02 \$A2.00+0.00. 
THEOREM 1. For a Banach Space $X$, the following are equivalent.

(i) $X$ has the Radon-Nikodym Property.

(ii) Every continuous weak ${ }^{\star}$ lower semicontinuous convex function on an open convex subset of $X^{\star}$ is Fréchet differentiable at the points of a dense $G_{\delta}$ subset of its domain.

(iii) Every continuous weak ${ }^{\star}$ lower semicontinuous convex function on an open convex subset of $X^{\star}$ is Gâteaux differentiable at a point of its domain with derivative in $\widehat{X}$.

(iv) Every continuous weak ${ }^{\star}$ lower semicontinuous gauge $p$ on $X^{\star}$ has a point $f \in X^{\star}$ with $\partial p(f) \subseteq \widehat{X}$.

\section{PROOF:}

That (i) $\Longrightarrow$ (ii) is Collier's result, [3, Theorem 1, p. 103]. It is obvious that (ii) $\Longrightarrow$ (iii) $\Longrightarrow$ (iv).

To prove (iv) $\Longrightarrow$ (i), consider $K$ a closed bounded convex subset of $X$. We may assume that $0 \in K$. Consider the continuous positive sublinear functional $p$ on $X^{\star}$ where

$$
p(f)=\sup \{f(x): x \in K\}
$$

As $p$ is the gauge of $K^{\circ} \equiv\left\{f \in X^{\star}: p(f) \leq 1\right\}$, it is weak ${ }^{\star}$ lower semicontinuous on $X^{\star}$.

From (iv) there exists an $f_{0} \in X^{\star}$ such that $\partial p\left(f_{0}\right) \subseteq \widehat{X}$. Now $\partial p\left(f_{0}\right)$ is an extreme subset of $\widehat{\widehat{K}}^{w^{\star}}$. Since $\partial p\left(f_{0}\right)$ is weak ${ }^{\star}$ compact, by the Krein-Milman Theorem $\partial p\left(f_{0}\right)$ has an extreme point $\widehat{x}_{0}$ which is then an extreme point of $\widehat{\widehat{K}}^{\boldsymbol{w}^{\star}}$.

Suppose that $x_{0} \notin K$. Then $x_{0}$ can be strongly separated from $K$ by a weakly closed hyperplane and so $\widehat{x}_{0}$ can be strongly separated from $\widehat{K}$ by a weak ${ }^{\star}$ closed hyperplane. But this contradicts $\widehat{x}_{0} \in \overline{\widehat{K}}^{w^{*}}$. So we conclude that $\widehat{K}$ contains an extreme point of $\widehat{\widehat{K}}^{w^{\star}}$ which, by the Proposition implies that $X$ has the Radon-Nikodym Property.

Bachir and Daniilidis point out that $c_{0}$, which does not have the Radon-Nikodym Property, does have the norm $\|\cdot\|_{1}$ on its dual $\ell_{1}$ which is generically Gâteaux differentiable with derivatives in $\ell_{\infty} \backslash \widehat{c}_{0}$. But it should also be noticed that there is no point of $\ell_{1}$ where the subdifferential of norm $\|\cdot\|_{1}$ lies in $\widehat{c}_{0}$.

It is worth expressing the equivalence (i) $\Longleftrightarrow$ (iv) of Theorem 1 in terms of set properties in the original space $X$.

Given a nonempty set $K$ in a normed linear space $X$, a subset $S$ of $K$ is said to 
be exposed by $f_{0} \in X^{\star}$ if for every $x \in S$,

$$
f_{0}(x)=\sup f_{0}(K)>f_{0}(y) \quad \text { for all } y \in K \backslash S \text {. }
$$

A slice of $K$ by $f_{0} \in X^{\star}$ is a subset of the form

$$
S\left(K, f_{0}, \delta\right) \equiv\left\{x \in K: f_{0}(x)>\sup f_{0}(K)-\delta\right\} \quad \text { for } \delta>0 .
$$

We say that $S$ is weakly exposed by $f_{0} \in X^{\star}$ if $S$ is exposed by $f_{0}$ and given a weak open neighbourhood $W$ of 0 , there exists a $\delta>0$ such that

$$
S\left(K, f_{0}, \delta\right) \subseteq S+W
$$

Lemma. Given a nonempty closed bounded convex set $K, 0 \in K$ in a normed linear space $X$, a subset $S$ of $K$ is weakly compact and weakly exposed by $f_{0} \in X^{\star}$ if and only if $\widehat{S}=\partial p\left(f_{0}\right)$ where $p$ is the continuous weak $k^{\star}$ lower semicontinuous gauge of the polar $K^{\circ}$.

Proof: Suppose $\widehat{S}=\partial p\left(f_{0}\right)$ but $S$ is not weakly exposed by $f_{0}$. Then there exists a weak neighbourhood $W$ of 0 and for each $n \in \mathbb{N}, x_{n} \in S\left(K, f_{0},(1 / n)\right) \backslash(S+W)$. Now $\left\{\widehat{x}_{n}\right\}$ has a weak ${ }^{\star}$ cluster point $F \in \widehat{\widehat{K}}^{\omega^{\star}}$ and $F \in \partial p\left(f_{0}\right)$. But then $F \in \widehat{K}$ and $\left\{x_{n}\right\}$ has a weak cluster point in $S$ which contradicts our supposition.

Conversely, suppose that $S$ is weakly compact and weakly exposed by $f_{0} \in X^{\star}$ but that there exists $F_{0} \in \partial p\left(f_{0}\right) \backslash \widehat{S}$. Since $\widehat{S}$ is convex and weak ${ }^{\star}$ compact we can strongly separate $F_{0}$ from $\widehat{S}$ by some $g \in X^{\star}$.

Then there exists a weak ${ }^{\star}$ open set $N \equiv\left\{F \in X^{\star \star}: F(g)>\alpha\right\}$ such that $\widehat{S} \subseteq N$ and $F_{0} \in\left\{F \in X^{\star \star}: F(g)<\alpha\right\}$. But also there exists a sequence of weak ${ }^{\star}$ open sets

$$
M_{n} \equiv\left\{F \in X^{\star \star}: F\left(f_{0}\right)>\sup \widehat{f}_{0}\left(\widehat{K}^{\omega^{\star}}\right)-\frac{1}{n}\right\} \cap\left\{F \in X^{\star \star}: F(g)<\alpha\right\} .
$$

and $F_{0} \in \partial p(f) \cap M_{n}$ for all $n \in \mathbb{N}$. Now the subdifferential mapping $f \mapsto \partial p(f)$ has the property that for any open subset $U$ of $X^{\star}$ and weak ${ }^{\star}$ open half-space $W$ in $X^{\star \star}$ where $\partial p(U) \cap W \neq \emptyset$ there exists a nonempty open subset $V$ of $U$ such that $\partial p(V) \subseteq W$. Using this and the extended Bishop-Phelps Theorem [8], we have for each $n \in \mathbb{N}$ there exists $\widehat{x}_{n} \in S\left(\overline{\widehat{K}}^{\star}, f_{0},(1 / n)\right) \cap M_{n}$. But then $\widehat{x}_{n} \in S\left(\widehat{K}, \widehat{f}_{0},(1 / n)\right) \backslash N$ which contradicts $S$ being weakly exposed by $f_{0}$.

Consequently we have a characterisation which generalises that of $[1$, Corollary 4 (i) $\Longleftrightarrow$ (iii)]. 
Theorem 2. A Banach space $X$ has the Radon-Nikodym Property if and only if every nonempty bounded closed convex set $K$ in $X$ has a subset which is weakly compact and weakly exposed.

A Banach space $X$ is an Asplund space if and only if its dual $X^{\star}$ has the RadonNikodym Property, [7, p. 82]. So our characterisations of the Radon-Nikodym Property imply extended characterisations of Asplund spaces.

Corollary. For a Banach space $X$, the following are equivalent.

(i) $X$ is an Asplund space.

(ii) Every continuous weak $k^{\star}$ lower semicontinuous gauge $p$ on $X^{\star \star}$ has a point $F \in X^{\star \star}$ with $\partial p(F) \subseteq \widehat{X}^{\star}$.

(iii) Every nonempty weak ${ }^{\star}$ compact convex set $K$ in $X^{\star}$ has a subset which is weakly compact and weakly exposed.

\section{REFERENCES}

[1] M. Bachir and A. Daniilidis, 'A dual characeristation of the Radon-Nikodym Property', Bull. Austral. Math. Soc. 62 (2000), 379-387.

[2] R. Bourgin, Geometric aspects of convex sets with the Radon-Nikodym Property, Lecture notes in Mathematics, 993 (Springer Verlag, Berlin, Heidelberg, New York, 1983).

[3] J. Collier, 'The dual of a space with the Radon-Nikodym Property', Pacific J. Math. 64 (1976), 103-106.

[4] J.R. Giles, 'Comparable differentiability characterisations of two classes of Banach spaces', Bull. Austral. Math. Soc. 56 (1997), 263-272.

[5] J.R. Giles, 'Comparable characterisations of two classes of Banach spaces by subdifferentials', Bull. Austral. Math. Soc. 58 (1998), 155-158.

[6] G. Godefroy, 'Metric characterisations of first Baire class linear forms and octahedral norms', Studia Math. 95 (1989), 1-15.

[7] R.R. Phelps, Convex functions, monotone operators and differentiability (2nd edition), Lecture notes in Mathematics 1364, (Springer Verlag, Berlin, Heidelberg, New York, 1993).

[8] R.R. Phelps, 'Weak ${ }^{\star}$ support points of convex sets in $E^{\star}$, Israel J. Math. 2 (1964), 177-182.

Department of Mathematics

The University of Newcastle

New South Wales 2308

Australia 